\title{
Study of Treatment Outcome in Adults with TFE-Related RCC
}

\author{
Ajaykumar Chandrabhan Singh ${ }^{1} \quad$ Mahendra Pal ${ }^{2} \quad$ Akhil Kapoor $^{3}$, Nandini Menon ${ }^{1} \quad$ Kumar Prabhash ${ }^{1}$ \\ Vanita Noronha ${ }^{1}$ Ganesh Bakshi ${ }^{2}$ Gagan Prakash² Santosh Menon ${ }^{4}$ Nilesh Sable $^{5}$ Devanshi Kalra ${ }^{6}$ \\ Sheetal Kulkarni ${ }^{6} \quad$ Amit Joshi ${ }^{1}$
}

${ }^{1}$ Department of Medical Oncology, Tata Memorial Hospital, Homi Bhabha National Institute, Mumbai, Maharashtra, India

2Department of Urologic Oncology, Tata Memorial Hospital, Homi Bhabha National Institute, Mumbai, Maharashtra, India

${ }^{3}$ Department of Medical Oncology, Mahamana Pandit Madan Mohan Malviya Cancer Center and Homi Bhabha Cancer Hospital (A Unit of Tata Memorial Center, Mumbai), Varanasi, Uttar Pradesh, India

${ }^{4}$ Department of Pathology, Tata Memorial Centre, Homi Bhabha National Institute, Mumbai, Maharashtra, India

\author{
${ }^{5}$ Department of Radiology, Tata Memorial Centre, Homi Bhabha \\ National Institute, Mumbai, Maharashtra, India \\ ${ }^{6}$ CRS, Tata Memorial Hospital, Homi Bhabha National Institute, \\ Mumbai, Maharashtra, India
}

Address for correspondence Amit Joshi, DM, Department of Medical Oncology, Tata Memorial Hospital, HBNI, HBB 1105, 11th floor, Mumbai, Maharashtra 400012, India (e-mail: dramitjoshi74@gmail.com).

South Asian J Cancer 2021;10:92-96.

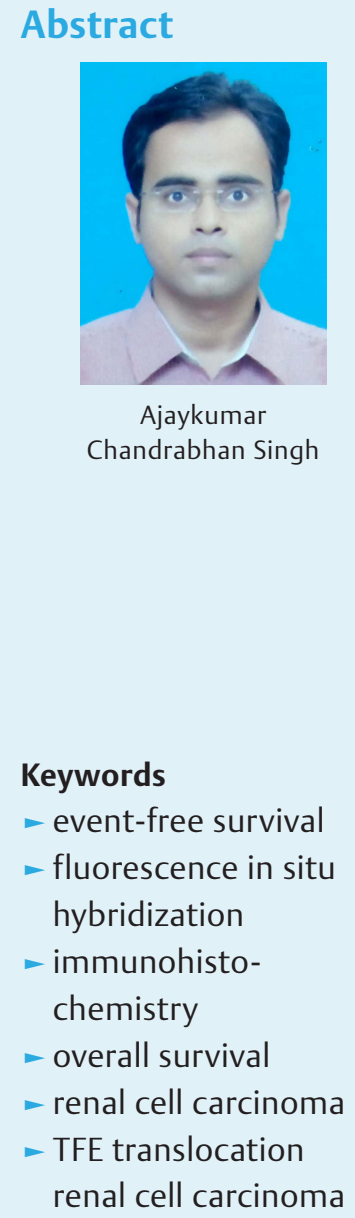

Introduction TFE Translocation renal cell carcinoma (TRCC) represents 1 to $5 \%$ of all cases of renal cell carcinoma, with the highest frequency among children and young adults. Management of these tumors is not very well defined in literature. Although in pediatric age group it has favorable prognosis, in adults it has an aggressive nature, with poor outcome. This is a retrospective analysis of treatment outcome in adult patient 18 years or above treated at our hospital between January 2013 and November 2018. Material and Methods Clinical and pathological data of 26 patients from a single institution diagnosed with TRCC between January 2013 and November 2018 were retrospectively reviewed. All cases of TRCC were confirmed with immunohistochemistry or fluorescence in situ hybridization. We analyzed our data of patients treated with surgery only or who progressed after surgery and treated with systemic therapy or who presented with upfront unresectable or metastatic disease treated with systemic therapy with respect to event-free survival (EFS) and overall survival (OS).

Results Between January 2013 and November 2018, 26 adult patients who were treated at our center were eligible for this analysis as per our criteria. Out of 26 patients, 25 patients had radical surgery after evaluation and 1 had metastatic disease who was started on systemic therapy. Out 25 patients who were treated with radical surgery, 16 patients progressed and they were started on systemic therapy except for 1 patient who defaulted. Median time to start systemic therapy among patient treated with curative nephrectomy was 13 months. Median EFS and median OS among overall population were 22 and 30 months, respectively. Among 16 patients who were treated with systemic therapy, median EFS to first-line therapy was 8 months and to second-line therapy was 2.5 months. Median OS was 17 months in patients treated with systemic therapy.

Conclusion TRCC is rare in adult population but carries significant risk of disease progression even after initial curative treatment with potential response to targeted therapy for short duration.

\section{DOI https://doi.org/10.1055/s-0041-1731264 ISSN 2278-330X}

How to cite this article: Singh A. C, Pal M, Kapoor A, et al. Study of Treatment Outcome in Adults with TFE-Related RCC. South Asian J Cancer 2021;10(2):92-96. (c) 2021. MedIntel Services Pvt Ltd.

This is an open access article published by Thieme under the terms of the Creative Commons Attribution-NonDerivative-NonCommercial-License, permitting copying and reproduction so long as the original work is given appropriate credit. Contents may not be used for commercial purposes, or adapted, remixed, transformed or built upon. (https://creativecommons.org/licenses/by-nc-nd/4.0/).

Thieme Medical and Scientific Publishers Private Ltd A-12, Second Floor, Sector -2, NOIDA -201301, India 


\section{Introduction}

Translocation carcinomas of the kidney were first described in children and adolescents and are usually considered indolent, even if diagnosed at an advanced stage in this population of patients. ${ }^{1} \mathrm{Xp} 11$ translocation renal cell carcinomas (TRCCs) have recently been included as a separate entity in the 2004 World Health Organization (WHO) classification of renal tumors. ${ }^{2}$ Now these tumors are grouped under heading of "MiT family TRCC ( $>$ Table 1 )."

The Xp11 TRCCs were first officially recognized in the 2004 WHO renal tumor classification, ${ }^{3}$ and harbor gene fusions involving TFE3 (transcription factor E3).

Although these tumors comprise at least one-third of pediatric RCCs, but incidence in adults are very less. ${ }^{4}$ Given the degree to which these tumors overlap morphologically with clear cell and/or papillary RCC, many cases of adult TRCC may be misclassified as clear cell or papillary RCC, and thus the true incidence of this entity may in fact be underestimated. ${ }^{5}$ In the past few years, several reports of adults with TRCC having an aggressive clinical course have emerged. ${ }^{6-8}$

Recent studies have shown that adult Xp11 TRCC is more common than originally perceived, making up anywhere from 1.6 to $5 \%$ of adult RCCs. ${ }^{9,10}$ Komai et $\mathrm{al}^{9}$ examined 443 consecutive adult Japanese patients from their institution with renal masses and observed a $1.6 \%$ incidence rate. ${ }^{9}$ Zhong et al $^{10}$ subsequently reported an incidence of $5 \%$ after examining 121 consecutive adult pathologic cases. In their study, they found 6 (5.0\%) of 121 of their patients possessed strong TFE3 immunohistochemistry (IHC) staining and harbored Xp11 translocations confirmed by dual-color break-apart fluorescence in situ hybridization (FISH).

Despite its relatively uncertain and occasionally indolent progression in children, Xp11 TRCC has been shown to possess an aggressive course in adults. ${ }^{6,11,12}$ Most patients diagnosed with Xp11 TRCC either present with metastasis or subsequently develop metastasis within 12 months of the initial presentation. ${ }^{4,6}$ Argani et al, ${ }^{4}$ who examined 28 cases, all of which were confirmed by TFE3 IHC, and found that one-third of patients with long-term follow-up died within 12 months.

To our knowledge, there are no established effective therapies for advanced/unresectable/recurrent/metastatic Xp11.2 TRCC. Therefore, we performed a retrospective analysis of adult patients with TRCC including early/advanced/metastatic/relapsed who were treated at our institution to assess the clinical features and treatment outcome of this particular RCC subtype in adults.

\section{Materials and Methods}

Patients aged 18 years and above with RCC including early, advanced, and metastatic who underwent clinical evaluation at Tata Memorial Hospital, Mumbai, Maharashtra, India, diagnosed as TRCC by either IHC or FISH and were treated with various modality including surgery or systemic therapy between January 2013 and November 2019 were the subjects of this retrospective review. A total of 26 patients were identified who meet these parameters. At the time of the presentation detailed history and physical examinations, as well as baseline laboratory parameters such as blood counts and chemistries, including lactate dehydrogenase, were taken, allowing them to be stratified into the recent prognostic groups by Heng et al. ${ }^{13}$ Data collected included standard pretreatment disease characteristics, baseline biochemical parameters, first date of treatment, date of disease progression, further line of treatment, and date of death or last follow-up.

\section{Statistical Analysis}

Median survival was defined as the time from the initiation of treatment to the date of death or censoring at the time of last follow-up. Event-free survival (EFS) was defined from the initiation of treatment to the date of disease progression, death, or censoring at the time of last follow- up. Survival distributions were estimated using the Kaplan-Meier method. ${ }^{14}$ All tests of statistical significance were two-sided. All data analyses were conducted using IBM SPSS Statistics for Windows, Version 23.0 (IBM Corp., Armonk, New York, Untied States).

Table 1 Cancers with Xp11 translocation/TFE3 gene fusions

\begin{tabular}{|l|l|l|l|}
\hline Neoplasm & Fusion & $\begin{array}{l}\text { Age range } \\
\text { in year }\end{array}$ & Translocation \\
\hline ASPS & ASPSCR1-TFE3 & $1-71$ & $\operatorname{der}(17)(\mathrm{X} ; 17)(\mathrm{p} 11.2 \mathrm{q} 25)$ \\
\hline RCC & ASPSCR1-TFE3 & $1-75$ & $\mathrm{t}(\mathrm{X} ; 17)(\mathrm{p} 11.2 ; \mathrm{q} 25)$ \\
\hline RCC & PRCC-TFE3 & $2-69$ & $\mathrm{t}(\mathrm{X} ; 1)(\mathrm{p} 11.2 ; \mathrm{q} 21)$ \\
\hline RCC & SFPQ-TFE3 & $3-68$ & $\mathrm{t}(\mathrm{X} ; 1)(\mathrm{p} 11.2 ; \mathrm{q} 34)$ \\
\hline RCC & NonO-TFE3 & 39 & $\mathrm{inv}(\mathrm{X})(\mathrm{p} 11.2 ; \mathrm{q} 12)$ \\
\hline RCC & CLTC-TFE3 & 14 & $\mathrm{t}(\mathrm{X} ; 17)(\mathrm{p} 11.2 ; \mathrm{q} 23)$ \\
\hline RCC & Unknown & 32 & $\mathrm{t}(\mathrm{X} ; 3)(\mathrm{p} 11.2 ; \mathrm{q} 23)$ \\
\hline RCC & Unknown & 77 & $\mathrm{t}(\mathrm{X} ; 10)(\mathrm{p} 11.2 ; \mathrm{q} 23)$ \\
\hline Xp11 PEComa & SFPQ-TFE3 and others & $9-55$ & $\mathrm{t}(\mathrm{X} ; 1)(\mathrm{p} 11.2 ; \mathrm{q} 34)$ and others \\
\hline Melanotic Xp11 translocation cancer & SFPQ-TFE3 and likely others & $11-45$ & $\mathrm{t}(\mathrm{X} ; 1)(\mathrm{p} 11.2 ; \mathrm{q} 34)$ and likely others \\
\hline Subset of epithelioid hemangioendothelioma & YAP1-TFE3 & $14-50$ & $\mathrm{t}(\mathrm{X} ; 11)(\mathrm{p} 11.2 ; \mathrm{q} 13)$ \\
\hline
\end{tabular}

Abbreviations: ASPS, alveolar soft part sarcoma; RCC, renal cell carcinoma. 


\section{Results}

Patient characteristics are summarized in - Table 2.

Twenty-six patients were included in this analysis. The median age at the time of the initiation of therapy was 30.5 years (range, 18-66 years). Out of 26 patients, 14 were females and 12 were males. At presentation the Eastern Cooperative Oncology Group performance status was 1 of all patients. The method of detection of TRCC was by IHC for TFE in 25 out of 26 patients, all showed diffuse staining for TFE, and in 1 patient by both, IHC for TFE which was focal, and later confirmed by FISH method. At presentation out of 26 patients, 25 patients underwent radical nephrectomy who were on evaluation and had either early or locally advanced stage disease. One patient presented with upfront metastatic disease who was started on systemic therapy. Out of 25 patients who had undergone curative nephrectomy 16 had relapse and 9 are still disease-free when this analysis is done. The median time to relapse was 13 months (range, 1-54 month). Out of 26 patients, 18 patients were stratified

Table 2 Patient characteristics $(\mathrm{N}=26)$

\begin{tabular}{|c|c|}
\hline Baseline characteristics & Number \\
\hline \multicolumn{2}{|l|}{ Gender } \\
\hline Male & 12 \\
\hline Female & 14 \\
\hline \multicolumn{2}{|l|}{ Age in years } \\
\hline $18-30$ & 13 \\
\hline $31-40$ & 7 \\
\hline $41-50$ & 4 \\
\hline $51-60$ & 1 \\
\hline$>60$ & 1 \\
\hline \multicolumn{2}{|l|}{ Performance status - ECOG } \\
\hline $0-1$ & 26 \\
\hline$\geq 2$ & 0 \\
\hline \multicolumn{2}{|l|}{ Method of detection } \\
\hline $\mathrm{IHC}$ & 25 \\
\hline FISH & 0 \\
\hline $\mathrm{IHC}+\mathrm{FISH}$ & 1 \\
\hline Cytogenetics & 0 \\
\hline \multicolumn{2}{|l|}{ Presentation } \\
\hline Resectable (early + LA) & 25 \\
\hline Unresectable (LA + metastatic) & 1 \\
\hline \multicolumn{2}{|l|}{ Treatment upfront on presentation } \\
\hline Curative nephrectomy & 25 \\
\hline Systemic therapy & 1 \\
\hline \multicolumn{2}{|l|}{ Progression after curative nephrectomy } \\
\hline Yes & 16 \\
\hline No & 9 \\
\hline
\end{tabular}

Abbreviations: ECOG, Eastern Cooperative Oncology Group; FISH, fluorescence in situ hybridization; $I H C$, immunohistochemistry; LA, locally advanced. according to the International Metastatic RCC Database Consortium criteria, into favorable group - 6, intermediate group -8 , and high-risk group - 4 . Eight patients did not have all the parameters to calculate the Heng's score. Sixteen of 25 patients who relapsed after nephrectomy, 5 relapsed locoregionally and 11 had distant relapse.

Out of 11 patients who had distant relapse, 2 had metastasis to lung and liver, 3 had metastasis to lung and brain, 1 had metastasis to liver, and 5 had metastasis to lung.

\section{Subsequent Therapies ( - Table 3)}

Seventeen patients received systemic therapy including 16 relapsed patients and 1 patient upfront. Among first-line therapy, 7 received sorafenib, 6 received sunitinib, 3 received pazopanib, and 1 patient defaulted before starting therapy. Among the 16 patients, 8 received second-line systemic therapy, 3 received everolimus, 1 thalidomide, 1 sunitinib, 1 axitinib, 1 nivolumab, and 1 nivolumab plus axitinib. Three patients later received third-line therapy, 2 received thalidomide and 1 cabozantinib. One patient further received fourth-line phase one drug and later fifth line as sunitinib.

\section{Treatment Outcome}

Among the 25 patients who underwent upfront nephrectomy, median EFS was 13 months. Median EFS and median overall survival (OS) among overall population was 22 and 30 months, respectively. Among 16 patients who were treated with systemic therapy, median EFS to first-line therapy was 8 months and to second-line therapy was 2.5 months. Median OS was 17 months among 16 patients who were treated with systemic therapy.

\section{Toxicity}

Among 16 patients who were treated with systemic therapy predominantly tyrosine kinase inhibitor (TKI) based,

Table 3 Systemic treatment details

\begin{tabular}{|c|l|}
\hline First line $(\boldsymbol{N}=\mathbf{1 6})$ & \\
\hline Sorafenib & 7 \\
\hline Sunitinib & 6 \\
\hline Pazopanib & 3 \\
\hline Defaulted & 1 \\
\hline Second line $(N=8)$ & \\
\hline Everolimus & 3 \\
\hline Axitinib & 1 \\
\hline Sunitinib & 1 \\
\hline Thalidomide & 1 \\
\hline Nivolumab & 1 \\
\hline Nivolumab + axitinib & 1 \\
\hline Third line $(N=3)$ & \\
\hline Thalidomide & 2 \\
\hline Cabozantinib & 1 \\
\hline Fourth + fifth line $(N=1)$ & \\
\hline Trial drug $\mathrm{f} / \mathrm{b}$ sunitinib & 1 \\
\hline
\end{tabular}


8 patients had grade $3 / 4$ toxicity, 4 had hand-foot syndrome (HFS), 2 had HFS and mucositis, 1 had HFS, mucositis, and diarrhea, and 1 had significant proteinuria and hypertension. Among patients who received further lines of therapy there was no any grade $3 / 4$ toxicity seen.

\section{Discussion}

As per our knowledge, this may be one of the largest data of treatment outcomes of patients with translocation-associated RCC which includes patient who presented with early, locally advanced, metastatic, and relapsed cases who were treated with different treatment modality which included surgical and different systemic therapies like TKIs and immunotherapy.

Although several targeted agents and immunotherapy have been approved for the treatment of advanced RCC, allowing oncologists to treat all RCC subtypes, clinical trials have for the most part been conducted in patients with clear cell histology (the most common subtype), with some limited experience reported in patients with nonclear cell subtypes such as papillary and chromophobe $\mathrm{RCC} .{ }^{15}$ Prior reports from the literature suggest that patients with Xp11.2 TRCC do not benefit from immunotherapy or chemotherapy, although these studies included $<5$ patients with advanced disease. To our knowledge, to date there has not been a reported case with a significant response to those therapies. ${ }^{8,9,16}$ In contrast, two case reports ${ }^{17,18}$ have shown that sunitinib may have significant activity in patients with TRCC.

In our study, median EFS and median OS among overall population was 22 and 30 months, respectively. Among 16 patients who were treated with systemic therapy, median EFS to first-line therapy was 8 months and to second-line therapy was 2.5 months. Median OS was 17 months among 16 patients who were treated with systemic therapy. Median time to progression among patient treated with curative nephrectomy was 13 months, which is almost same as reported by Argani et $\mathrm{al}^{4}$ in their study in which one-third of patients progressed within 12 months of curative treatment.

A multicenter study from several French centers reported on 21 patients with metastatic Xp11.2 RCC who were treated with vascular endothelial growth factor (VEGF) and mechanistic target of rapamycin-targeted therapies. ${ }^{19}$ Patients treated with sunitinib were found to have a median progression-free survival (PFS) of 8.2 months if they received therapy before treatment with sunitinib, and 11 months if they were previously treatment-naive. Patients treated with sorafenib had a median PFS of 6 months, whereas patients treated with temsirolimus had a median PFS of 3 months. Seven patients (33\%) experienced objective responses. All patients treated with sunitinib and 1 patient treated with temsirolimus achieved responses. With a median follow-up of 19 months, the median OS was reported to be 27 months. ${ }^{19}$ The efficacy data in the French series are somewhat higher than in the current report, which could be because of case selection bias or the fact that they did not restrict their study to adult patients. In fact, 5 patients in the French series (24\%) were aged $<18$ years. As shown in multiple reports, patients with adult Xp11.2 RCC tend to have a more aggressive disease course than their pediatric counterparts. ${ }^{6.8}$

Another recent study ${ }^{20}$ demonstrated that VEGF-targeted therapy can be of benefit in adults with metastatic Xp11.2 RCC, as evidenced by a response rate of $20 \%$ and a median PFS of 7.1 months. However, based on the small number of patients in the study and the finding that responses occurred with three different drugs, it is impossible to make definitive conclusions regarding the best VEGF-targeted agent for patients with this disease. ${ }^{20}$

In our study, the incidence of TRCC was almost equal among male and female, unlike other reports which show female predominance. Median age of presentation in our study was 30.5 years among adult population which was same as other reports.

As compared with pediatrics population which remain disease free for long, may be cured also, after radical nephrectomy for early and locally advanced disease; among adults in our study out of 25 patients who were treated with curative nephrectomy, 16 (64\%) patients had relapsed with median EFS of 13 month only, which was also same as other studies which have shown that adult patients with this disease relapse within a year after initial curative surgery. Our study has also shown that patients who relapsed and had metastatic disease can still be treated with TKI therapy with significant benefit, that is, median EFS of 8 months, but EFS to second-line systemic therapy was short, that is, 2.5 months. Although two of our patients had received immunotherapy in second-line setting and had significant benefit with immunotherapy, this number is very small to make any definitive conclusion.

\section{Conclusion}

TRCC is rare but we need to keep this differential in our mind when we come across any young patient who present with RCC. TRCC has more aggressive course in adult population compared with pediatric patients. More collaborative studies are required to exactly define the optimum therapies in this patient population.

\section{Note}

The study was conducted in accordance with the Declaration of Helsinki and the International Conference on Harmonization Guidelines for Good Clinical Practice.

\section{Conflict of Interest}

None declared.

\section{References}

1 Argani P, Ladanyi M. Translocation carcinomas of the kidney. Clin Lab Med 2005;25(2):363-378

2 Eble J, Sauter G, Epstein JI, WHO Classification of Tumours. Tumours of the Genitourinary and Male Genital Organs. Lyon: IARC Press; 2004

3 Argani P, Ladanyi M, Renal carcinomas associated with Xp11.2 translocations/TFE3 gene fusions. In: Eble JN, Sauter G, Epstein J, et al, eds. Pathology and Genetics of Tumors of 
the Urinary System \& Male Genital Organs. Lyon, France: IARC; 2004 37-38

4 Argani P, Olgac S, Tickoo SK, et al. Xp11 translocation renal cell carcinoma in adults: expanded clinical, pathologic, and genetic spectrum. Am J Surg Pathol 2007;31(8):1149-1160

5 Argani P, Lal P, Hutchinson B, Lui MY, Reuter VE, Ladanyi M. Aberrant nuclear immunoreactivity for TFE3 in neoplasms with TFE3 gene fusions: a sensitive and specific immunohistochemical assay. Am J Surg Pathol 2003;27(6):750-761

6 Meyer PN, ClarkJI, Flanigan RC, Picken MM.Xp11.2 translocation renal cell carcinoma with very aggressive course in five adults. Am J Clin Pathol 2007;128(1):70-79

7 Rais-Bahrami S, Drabick JJ, De Marzo AM, et al. Xp11 translocation renal cell carcinoma: delayed but massive and lethal metastases of a chemotherapy-associated secondary malignancy. Urology 2007;70(1):178.e3-178.e6

8 Armah HB, Parwani AV. Renal cell carcinoma in a 33-year-old male with an unusual morphology and an aggressive clinical course: possible Xp11.2 translocation. Pathology 2008;40(3):306-308

9 Komai Y, Fujiwara M, Fujii Y, et al. Adult Xp11 translocation renal cell carcinoma diagnosed by cytogenetics and immunohistochemistry. Clin Cancer Res 2009;15(4):1170-1176

10 Zhong M, De Angelo P, Osborne L, et al. Translocation renal cell carcinomas in adults: a single-institution experience. Am J Surg Pathol 2012;36(5):654-662

11 Parikh J, Coleman T, Messias N, Brown J. Temsirolimus in the treatment of renal cell carcinoma associated with Xp11.2 translocation/TFE gene fusion proteins: a case report and review of literature. Rare Tumors 2009;1(2):e53
12 Numakura K, Tsuchiya N, Yuasa T, et al. A case study of metastatic Xp11.2 translocation renal cell carcinoma effectively treated with sunitinib. Int J Clin Oncol 2011;16(5):577-580

13 Heng DY, Xie W, Regan MM, et al. Prognostic factors for overall survival in patients with metastatic renal cell carcinoma treated with vascular endothelial growth factor-targeted agents: results from a large, multicenter study. J Clin Oncol 2009;27(34):5794-5799

14 Kaplan E, Meier P. Nonparametric estimation from incomplete observations. J Am Stat Assoc 1958;53:457-481

15 NCCN Guidelines. Kidney Cancer. Version 1. 2021;MS9-MS26

16 Camparo P, Vasiliu V, Molinie V, et al. Renal translocation carcinomas: clinicopathologic, immunohistochemical, and gene expression profiling analysis of 31 cases with a review of the literature. Am J Surg Pathol 2008;32(5):656-670

17 Choueiri TK, Mosquera JM, Hirsch MS. A case of adult metastatic Xp11 translocation renal cell carcinoma treated successfully with sunitinib. Clin Genitourin Cancer 2009;7(3):E93-E94

18 Pwint TP, Macaulay V, Roberts IS, Sullivan M, Protheroe A. An adult Xp11.2 translocation renal carcinoma showing response to treatment with sunitinib. Urol Oncol 2011;29(6):821-824

19 Malouf GG, Camparo P, Oudard S, et al. Targeted agents in metastatic Xp11 translocation/TFE3 gene fusion renal cell carcinoma (RCC): a report from the Juvenile RCC Network. Ann Oncol 2010;21(9):1834-1838

20 Choueiri TK, Lim ZD, Hirsch MS, et al. Vascular endothelial growth factor-targeted therapy for the treatment of adult metastatic Xp11.2 translocation renal cell carcinoma. Cancer 2010;116(22):5219-5225 